\title{
P119 Carotid Stiffness is Strongly Associated with Skin Microvascular Dysfunction in Normotensives and Newly-diagnosed Hypertensives
}

\author{
Antonios Lazaridis*, Eugenia Gkaliagkousi, Nikolaos Koletsos, Eleni Gavriilaki, Panagiota Anyfanti, \\ Barbara Nikolaidou, Areti Triantafyllou, Konstantina Dipla, Stella Douma
}

Aristotle University of Thessaloniki, Thessaloniki, Greece

\section{ABSTRACT}

Background/Objectives: Skin microcirculation represents an easily accessible window reflecting generalized microvascular dysfunction. It has been correlated with alterations in several microvascular beds, though evidence regarding its association with macrovascular damage is scarce. Carotid artery stiffening is a surrogate marker of macrovascular damage that has been significantly associated with incident stroke and cardiovascular mortality. In this study, we investigated the association between skin microcirculation and carotid stiffness in a mixed population of hypertensive and normotensive individuals.

Methods: Untreated newly-hypertensive and normotensive individuals without major cardiovascular comorbidities were included in the study. Blood pressure (BP) parameters were recorded with the Mobil-O-Graph NG (IEM, Stolberg, Germany) device. Carotid measurements were performed using a high-resolution echo-tracking system (Prosound Alpha 7, Aloka, Japan). The beta index $(\beta)$ was automatically calculated and averaged for the right and left common carotid artery values. Skin microcirculation was assessed under standardized conditions using laser speckle contrast analysis (PeriCam PSI NR, Perimed, Sweden) in conjunction with post-occlusive reactive hyperemia protocol. Results are expressed as a percentage increase between baseline and peak perfusion (\%).

Results: We studied 101 individuals including 70 newly diagnosed, untreated essential hypertensives aged $49.6 \pm 9.4$ and 31 healthy normotensive individuals. The $\beta$ index demonstrated a significant negative correlation with the percentage increase between baseline and peak skin microvascular perfusion $(r=-0.251, p=0.01)$.

Conclusion: In a population of newly-diagnosed, untreated essential hypertensive and normotensive individuals, endothelial function of skin microcirculation is inversely associated with carotid stiffness, an emerging tool in cardiovascular risk prediction.

(C) 2019 Association for Research into Arterial Structure and Physiology. Publishing services by Atlantis Press International B.V. This is an open access article distributed under the CC BY-NC 4.0 license (http://creativecommons.org/licenses/by-nc/4.0/). 